\title{
Knowledge and Perception of Pharmacy Students about Generic Medicine in India: Web-based Cross-sectional Study
}

\author{
Amar Prashad Chaudhary", Adna Nelson K, Narayan Sah Sonar, Jamuna TR \\ Department of Pharmacy Practice, Mallige College of Pharmacy, Rajiv Gandhi University of Health Sciences, Bangalore, Karnataka, INDIA.
}

\begin{abstract}
Background: Globally, India being the largest provider of generic drugs, the students of all cadres of pharmacy need to have proper knowledge about generic drugs and their significance in the market, as well as in the lives of the consumers of the country. Objectives: The principal aim of this study is to compare and comprehend the knowledge and perceptions of B.Pharm, M.Pharm and Pharm.D students regarding generic medications. Methods: A cross-sectional web-based investigation was conducted for this study in September 2020. For this study, a semi-structured questionnaire of 22 items with four sections was constructed. They are the social demographic section, overall knowledge of generic medicine section, safety and efficacy of generic medicine section and the perception section. For this study, a quantitative analysis was carried out. Results: Significant difference exists in knowledge among these cadres regarding the marketing of generic medicine after the expiry of the patent right of innovator drug with a $P$-value of $<0.01^{* *}$. It is found that there exists a difference in knowl-
\end{abstract}

edge regarding efficacy, adverse effects, and safety of generic medicine among these cadres with a $P$-value less than $<0.001^{* * *}$. While comparing the perception of these cadres about the generic drug, there is a statistically significant vast difference. Conclusion: This study finds a significant disparity in knowledge and perceptions of generic medications across B.Pharm, M.Pharm and Pharm.D students.

Key words: B.Pharm, M.Pharm and Pharm.D, Generic medicine, Branded medicine, Pharmacy.

Correspondence

Dr. Amar Prashad Chaudhary,

Department of Pharmacy Practice, Mallige College of Pharmacy, Rajiv Gandhi University of Health Sciences, Bangalore-560090, Karnataka, INDIA.

Email id: pamar419@gmail.com

DOI: 10.5530/jyp.2021.13.100

\section{INTRODUCTION}

According to the WHO, about half of Asia and Africa's population lacks access to essential medications. India's situation is not much better, with health care spending accounting for only $1.2 \%$ of GDP and a per capita health expenditure of USD 160, well below the OECD (Organization for Economic Cooperation and Development) average of USD 3484. ${ }^{1}$ Drug prices are projected to account for two-thirds of total healthcare costs. According to Vandana et al., medicines are out of reach for economically disadvantaged people in India. ${ }^{2}$ One of the reasons for the lower economic segment of the population's lack of access to drugs is the exorbitant cost of branded medicines. To meet drug demand and reduce budgetary expenditure on drugs, the marketing of generic medicines is a viable alternative that is $20 \%$ to $90 \%$ less expensive than the brand-name counterpart. ${ }^{3,4}$

The pharmacist is critical for generic medicine promotion because they are directly involved in the formulation and dispensing of the medication. Pharmacists' clinical activities have advanced significantly over the last decade to ensure the appropriate and economical use of medications. ${ }^{4}$ However, successful generic medicine utilization is only achievable when pharmacists receive proper training in generic drugs as part of their degrees.

In India, a variety of pharmacy degrees are available, including the Diploma in Pharmacy (D.Pharm), the Bachelor of Pharmacy (B.Pharm), the Master of Pharmacy (M.Pharm), the Master of Science in Pharmacy [MS(Pharm)], and the Master of Technology in Pharmacy [MTech (Pharm)], as well as the Doctor of Pharmacy (Pharm.D) and doctor of philosophy in pharmacy $(\mathrm{PhD})$. The majority of students participate in B. Pharm and M.Pharm programs, which are primarily focused on the pharmaceutical industry. ${ }^{5}$ Students are increasingly interested in the
Pharm.D degree, which was introduced in 2008 as a clinically oriented pharmacy degree. These degrees include generic medicine subjects in their curricula, but they do not cover every facet of the field.

To the best of the author's knowledge, no similar research has been conducted in India to compare the knowledge and perception of generic medicine among diverse pharmacy cadres (B.Pharm, Pharm.D, and M.Pharm). As a result, the purpose of this study is to fill this knowledge gap by comparing B.Pharm, Pharm.D, and Mpharm students' understanding and perceptions of generic medicine.

\section{MATERIALS AND METHODS}

\section{Study design and study period}

In September 2020, a six-month cross-sectional web-based survey was conducted. This study engaged pharmacy students from Bangalore and other regions of South India who had access to the internet and were active on social media. This study adhered strictly to the Strengthening the Reporting of Observational Studies in Epidemiology (STROBE) standards for reporting. ${ }^{6,7}$

\section{Development of questionnaire}

A semi-structured questionnaire comprising of 22 items and divided into four sections was constructed for this investigation. The first segment featured four questions designed to elicit information about the participants' socio-demographic characteristics. Three sections contained a six-item multiple-choice questionnaire: general knowledge, knowledge about quality, safety, and efficacy, and perception. Strongly agree, agree, neutral, disagree, and strongly disagree were the options. All of these 
questions were developed utilizing a variety of research articles, books, fact sheets, and information booklets. The questionnaire was designed to ascertain students' fundamental knowledge and perceptions of generic medicines and their importance, particularly in the context of India.

\section{Validity and reliability test}

The initial questionnaire was validated by subject experts comprised of professors and other faculty members from several pharmacy institutions in Karnataka for correctness, clarity, appropriateness, and jargon use. This validation was conducted using both the face and content validity approach. The split-half method (for assessing internal reliability) and the test-retest method (for assessing external reliability) were used to evaluate the updated questionnaire's reliability. ${ }^{8,9}$ A pilot study with 20 individuals was done to conduct these reliability tests. In the Split-half reliability test, it was discovered that Spearman's brown coefficient of 0.7 correlates the responses to two halves of questions. The test-retest reliability was determined by administering the questionnaire twice, one week apart on the first and second attempts. In the test-retest reliability analysis, an intraclass correlation of 0.8 was discovered. Correlation coefficients greater than or equal to 0.7 indicate reliability. ${ }^{10}$

\section{Sample size}

The survey sample size was determined using the Raosoft sample size calculator. ${ }^{11}$ Minimum student involvement of 377 was required to achieve a $95 \%$ confidence interval and a $5 \%$ margin of error for the population distribution of 20000 students at a $50 \%$ response rate. As a result, we obtained the participation of 502 students for this study.

\section{Selection of students}

To assure the study's quality, participants were chosen based on their exposure to generic medicine in their syllabus. This study's criteria were established by an exhaustive analysis of the curriculums of several pharmacy courses and consultations with professors and faculty members at various pharmacy colleges. Finally, this study permitted the participation of third- and fourth-year B.Pharm (Bachelor of Pharmacy) students, third-, fourth-, fifth-, and sixth-year Pharm.D (Doctor of Pharmacy) students, and M.Pharm (Master of Pharmacy) students.

\section{Distribution of questionnaire}

Following the validation and reliability tests, the final revised questionnaire was created using Google Forms and sent to undergraduate and graduate scholars via social media platforms, including Facebook, Messenger, Telegram, and WhatsApp. Volunteers were asked to participate in the study by filling out a questionnaire in a Google form with no time constraints. The participant's name was recorded, and the Google feature was limited to one submission to avoid duplicate fillings of the Google form.

\section{Ethical consideration}

This study has been approved by the Mallige College of Pharmacy Research Review Board (MCP/RRB/002/20-21). The purpose of this study was described to the participants. They were requested to submit their consent of voluntary willingness before participating in this survey. The procedures utilized for this survey were as per the declaration of Helsinki 1964 and its later amendment. ${ }^{12}$ Furthermore, the Checklist for Reporting Results of Internet E-Surveys (CHERRIES) guidelines was strictly followed for performing and reporting this internet survey. ${ }^{13}$

\section{Statistical Analysis}

The data collected were uploaded to Microsoft Excel (Microsoft Corporation) and was analyzed for the correctness of the data. ${ }^{14}$ The statistical analysis was performed using IBM SPSS software, version 25 (IBM Cooperation). ${ }^{15}$ The difference in response to questions related to knowledge and perception were compared between different pharmacy cadres statistically, using the non-parametric Kruskal-Wallis test. The test was performed considering a $P$-value of less than .05 significant.

\section{RESULTS}

\section{Socio-demographic Details}

Table 1 summarizes the socio-demographic characteristics of the volunteers. The questionnaire was completed by 502 pharmacy students. Out of 502 pupils, 222 (44\%) were male, and 280 (56\%) were female. 268 students $(53.3 \%)$ who volunteered for the survey are between the ages of $18-21,216$ students $(43.02 \%)$ are between the ages of $22-25$, and only 18 students (3.5\%) are between the ages of 26 and above. Of the 502 students, 247 (49\%) were enrolled in Pharm.D, 211 (42\%) in B.Pharm, and 44 (9\%) in M.Pharm.

\section{Overall knowledge of generic medicine}

The overall knowledge of these cadres about generic medicine is represented in Table 2. It is discovered that these cadres have a statistically significant difference in their grasp of bioequivalence of generic with branded medicines, with a $P$-value smaller than $.001^{\star * *}$. At the same time, the majority of students across all of these cadres agree that they desire greater information about how bioequivalence testing for generic drugs is conducted, and so no statistically significant difference was discovered. There is disagreement among students in these cadres about whether the composition, dose and indication of generic medicines are equivalent to those of branded drugs and whether manufacturing of the generic medicine begins after the innovator drug's patent rights expire with a P-value of less than $.05^{\star}$ and $.01^{\star *}$, respectively. Additionally, these cadres continue to lack knowledge that generic medicines are more affordable than branded drugs and that increased use of generic medicines in India helps to lower healthcare spending by less than $.01^{* *}$ and $.001^{* * *}$, respectively.

\section{Safety and efficacy of generic medicine}

The knowledge of various pharmacy cadres regarding the safety and efficacy of generic medications is summarized in Table 3. This section discovered a difference in reaction between these cadres about the effectiveness and adverse effects of generic medications when compared to branded medications with a $P$-value smaller than $.001^{* * *}$. Whereas there seems to have the same level of knowledge and confusion among

\begin{tabular}{cc} 
Table 1: Socio-Demographic details. \\
\hline Socio-Demographic details & Participants Distribution $(\boldsymbol{n}=502)$ \\
\hline Age (years) & $268(53.3 \%)$ \\
$18-21$ & $216(43.02 \%)$ \\
$22-25$ & $12(2.3 \%)$ \\
$26-29$ & $06(1.19 \%)$ \\
$\geq 30$ & \\
Gender & $222(44 \%)$ \\
Male & $280(56 \%)$ \\
Female & \\
Degree Enrolled & $247(49 \%)$ \\
Pharm.D & $211(42 \%)$ \\
B.Pharm & $44(9 \%)$ \\
M.Pharm &
\end{tabular}


Table 2: summarizes the overall knowledge of pharmacy students regarding generic medicines according to their degree program.

\begin{tabular}{|c|c|c|c|c|c|c|c|c|}
\hline S. No & Questionnaire & Degree Enrolled & $\begin{array}{l}\text { Strongly } \\
\text { Agree } \\
(\%)\end{array}$ & $\begin{array}{c}\text { Agree } \\
(\%)\end{array}$ & $\begin{array}{l}\text { Neutral } \\
(\%)\end{array}$ & $\begin{array}{l}\text { Disagree } \\
\text { (\%) }\end{array}$ & $\begin{array}{l}\text { Strongly } \\
\text { Disagree } \\
\text { (\%) }\end{array}$ & $P$-value \\
\hline \multirow{3}{*}{1} & \multirow{3}{*}{$\begin{array}{l}\text { Generic medicines are inexpensive } \\
\text { compared to branded medicines. }\end{array}$} & B.Pharm $(\mathrm{N}=194)$ & $\begin{array}{c}77 \\
(40 \%)\end{array}$ & $\begin{array}{c}97 \\
(50 \%)\end{array}$ & $\begin{array}{c}13 \\
(7 \%)\end{array}$ & $\begin{array}{c}2 \\
(1 \%)\end{array}$ & $\begin{array}{c}5 \\
(2 \%)\end{array}$ & \multirow{3}{*}{0.003} \\
\hline & & M.Pharm (N=40) & $\begin{array}{c}27 \\
(68 \%)\end{array}$ & $\begin{array}{c}11 \\
(27 \%)\end{array}$ & $\begin{array}{c}1 \\
(2.5 \%)\end{array}$ & $\begin{array}{c}1 \\
(2.5 \%)\end{array}$ & $\begin{array}{c}0 \\
(0 \%)\end{array}$ & \\
\hline & & Pharm-D $(\mathrm{N}=220)$ & $\begin{array}{c}75 \\
(34 \%)\end{array}$ & $\begin{array}{c}90 \\
(41 \%)\end{array}$ & $\begin{array}{c}40 \\
(18 \%)\end{array}$ & $\begin{array}{c}4 \\
(1.8 \%)\end{array}$ & $\begin{array}{c}8 \\
(3.6 \%)\end{array}$ & \\
\hline \multirow{3}{*}{2} & \multirow{3}{*}{$\begin{array}{l}\text { A generic medicine is bioequivalent to a } \\
\text { branded medicine. }\end{array}$} & B.Pharm (N=193) & $\begin{array}{c}19 \\
(9.8 \%)\end{array}$ & $\begin{array}{c}117 \\
(60.6 \%)\end{array}$ & $\begin{array}{c}41 \\
(21.2 \%)\end{array}$ & $\begin{array}{c}11 \\
(5.6 \%)\end{array}$ & $\begin{array}{c}5 \\
(2.5 \%)\end{array}$ & \multirow{3}{*}{.000} \\
\hline & & M.Pharm (N=40) & $\begin{array}{c}16 \\
(40 \%)\end{array}$ & $\begin{array}{c}20 \\
(50 \%)\end{array}$ & $\begin{array}{c}1 \\
(2.5 \%)\end{array}$ & $\begin{array}{c}2 \\
(5 \%)\end{array}$ & $\begin{array}{c}1 \\
(2.5 \%)\end{array}$ & \\
\hline & & Pharm-D $(\mathrm{N}=218)$ & $\begin{array}{c}59 \\
(27 \%)\end{array}$ & $\begin{array}{c}104 \\
(47.7 \%)\end{array}$ & $\begin{array}{c}37 \\
(17 \%)\end{array}$ & $\begin{array}{c}17 \\
(7.8 \%)\end{array}$ & $\begin{array}{c}1 \\
(0.4 \%)\end{array}$ & \\
\hline \multirow{3}{*}{3} & \multirow{3}{*}{$\begin{array}{l}\text { Composition, dose, and indications } \\
\text { of generic medicines are equivalent to } \\
\text { branded medicines. }\end{array}$} & B.Pharm $(\mathrm{N}=192)$ & $\begin{array}{c}35 \\
(18.2 \%)\end{array}$ & $\begin{array}{c}94 \\
(49 \%)\end{array}$ & $\begin{array}{c}39 \\
(20.3 \%)\end{array}$ & $\begin{array}{c}22 \\
(11.4 \%)\end{array}$ & $\begin{array}{c}2 \\
(1 \%)\end{array}$ & \multirow{3}{*}{0.013} \\
\hline & & M.Pharm (N=38) & $\begin{array}{c}15 \\
(39.4 \%)\end{array}$ & $\begin{array}{c}16 \\
(42.1 \%)\end{array}$ & $\begin{array}{c}5 \\
(13.1 \%)\end{array}$ & $\begin{array}{c}1 \\
(2.6 \%)\end{array}$ & $\begin{array}{c}1 \\
(2.6 \%)\end{array}$ & \\
\hline & & Pharm-D $(\mathrm{N}=221)$ & $\begin{array}{c}62 \\
(28 \%)\end{array}$ & $\begin{array}{c}95 \\
(43 \%)\end{array}$ & $\begin{array}{c}40 \\
(18.1 \%)\end{array}$ & $\begin{array}{c}23 \\
(10.4 \%)\end{array}$ & $\begin{array}{c}1 \\
(0.4 \%)\end{array}$ & \\
\hline \multirow{3}{*}{4} & \multirow{3}{*}{$\begin{array}{l}\text { A generic medicine is only manufactured } \\
\text { after the end of the patent of the } \\
\text { innovator drug }\end{array}$} & B.Pharm $(\mathrm{N}=189)$ & $\begin{array}{c}32 \\
(16.9 \%)\end{array}$ & $\begin{array}{c}69 \\
(36.5 \%)\end{array}$ & $\begin{array}{c}38 \\
(20.1 \%)\end{array}$ & $\begin{array}{c}42 \\
(22.2 \%)\end{array}$ & $\begin{array}{c}8 \\
(4.2 \%)\end{array}$ & \multirow{3}{*}{0.002} \\
\hline & & M.Pharm (N=39) & $\begin{array}{c}18 \\
(46.1 \%)\end{array}$ & $\begin{array}{c}11 \\
(28.2 \%)\end{array}$ & $\begin{array}{c}5 \\
(12.8 \%)\end{array}$ & $\begin{array}{c}5 \\
(12.8 \%)\end{array}$ & $\begin{array}{c}0 \\
(0 \%)\end{array}$ & \\
\hline & & Pharm-D $(\mathrm{N}=218)$ & $\begin{array}{c}51 \\
(23.3 \%)\end{array}$ & $\begin{array}{c}57 \\
(26.1 \%)\end{array}$ & $\begin{array}{c}50 \\
(22.9 \%)\end{array}$ & $\begin{array}{c}47 \\
(21.5 \%)\end{array}$ & $\begin{array}{c}13 \\
(5.9 \%)\end{array}$ & \\
\hline \multirow{3}{*}{5} & \multirow{3}{*}{$\begin{array}{l}\text { Additional information is required on } \\
\text { how bioequivalence tests are carried out } \\
\text { for generic medicines }\end{array}$} & B.Pharm(N=191) & $\begin{array}{c}60 \\
(31.4 \%)\end{array}$ & $\begin{array}{c}102 \\
(53.4 \%)\end{array}$ & $\begin{array}{c}25 \\
(13 \%)\end{array}$ & $\begin{array}{c}3 \\
(1.5 \%)\end{array}$ & $\begin{array}{c}1 \\
(0.5 \%)\end{array}$ & \multirow{3}{*}{0.457} \\
\hline & & M.Pharm (N=39) & $\begin{array}{c}8 \\
(20.5 \%)\end{array}$ & $\begin{array}{c}24 \\
(61.5 \%)\end{array}$ & $\begin{array}{c}6 \\
(15.3 \%)\end{array}$ & $\begin{array}{c}1 \\
(2.5 \%)\end{array}$ & $\begin{array}{c}0 \\
(0 \%)\end{array}$ & \\
\hline & & Pharm-D $(\mathrm{N}=220)$ & $\begin{array}{c}70 \\
(31.8 \%)\end{array}$ & $\begin{array}{c}116 \\
(52.7 \%)\end{array}$ & $\begin{array}{c}23 \\
(10.4 \%)\end{array}$ & $\begin{array}{c}10 \\
(4.5 \%)\end{array}$ & $\begin{array}{c}1 \\
(0.4 \%)\end{array}$ & \\
\hline \multirow{3}{*}{6} & \multirow{3}{*}{$\begin{array}{l}\text { More usage of generic medicines in India } \\
\text { supports in reducing the country's health } \\
\text { care spending }\end{array}$} & B.Pharm $(\mathrm{N}=191)$ & $\begin{array}{c}45 \\
(23.5 \%)\end{array}$ & $\begin{array}{c}85 \\
(44.5 \%)\end{array}$ & $\begin{array}{c}30 \\
(15.7 \%)\end{array}$ & $\begin{array}{c}22 \\
(11.5 \%)\end{array}$ & $\begin{array}{c}9 \\
(4.7 \%)\end{array}$ & \multirow{3}{*}{.000} \\
\hline & & M.Pharm (N=39) & $\begin{array}{c}22 \\
(56.4 \%)\end{array}$ & $\begin{array}{c}10 \\
(25.6 \%)\end{array}$ & $\begin{array}{c}5 \\
(12.8 \%)\end{array}$ & $\begin{array}{c}2 \\
(5.1 \%)\end{array}$ & $\begin{array}{c}0 \\
(0 \%)\end{array}$ & \\
\hline & & Pharm-D $(\mathrm{N}=220)$ & $\begin{array}{c}96 \\
(43.6 \%)\end{array}$ & $\begin{array}{c}74 \\
(33.6 \%)\end{array}$ & $\begin{array}{c}23 \\
(10.4 \%)\end{array}$ & $\begin{array}{c}24 \\
(10.9 \%)\end{array}$ & $\begin{array}{c}3 \\
(1.3 \%)\end{array}$ & \\
\hline
\end{tabular}

Kruskal-Wallis test $P$-value $<.05^{*}, P$-value $<.01^{* *}$ and $P$-value $<.001^{* * *}$ is significant.

students of these cadres regarding the superiority of branded medicine over generic medicine in sophisticated safety standards. $(P$-value $=0.506)$. There is nevertheless a statistically significant difference in response about the generic medicine's quality, safety, and pharmacokinetics between these cadres with a $p$-value smaller than $.001^{* * *}$.

\section{Perception of students about generic medicine}

The perception of these cadres regarding generic medicine is summarized in Table 4. About $88.7 \%$ of B.Pharm students agree that patient and doctors should be provided sufficient knowledge regarding generic drugs whereas more than $90 \%$ of M.Pharm and Pharm.D agrees on the same statement $(P$-value $<0.05)$. The perception among these cadres about the influence of pharmaceutical companies by advertisement on the usage of branded medicine differs with $P$-value less than 0.001 . In addition, there remains a difference in ease of remembrance of drug's therapeutic class using generic names with a $P$-value less than 0.001 . About $81 \%$ of B.Pharm students and $82 \%$ of M.Pharm students agree they need more information on the safety and efficacy of generic medicine. In contrast, about $85 \%$ of Pharm.D agree on the topic. 
Table 3: Summarizes knowledge regarding the safety and efficacy of generic medicines according to their degree program.

\begin{tabular}{|c|c|c|c|c|c|c|c|c|}
\hline S. No & Questionnaire & $\begin{array}{l}\text { Degree } \\
\text { Enrolled }\end{array}$ & $\begin{array}{c}\text { Strongly } \\
\text { Agree } \\
(\%)\end{array}$ & $\begin{array}{l}\text { Agree } \\
(\%)\end{array}$ & $\begin{array}{l}\text { Neutral } \\
(\%)\end{array}$ & $\begin{array}{c}\text { Disagree } \\
\text { (\%) }\end{array}$ & $\begin{array}{c}\text { Strongly } \\
\text { Disagree } \\
\text { (\%) }\end{array}$ & $P$-value \\
\hline \multirow{3}{*}{1} & & $\begin{array}{l}\text { B.Pharm } \\
(\mathrm{N}=180)\end{array}$ & $\begin{array}{c}14 \\
(7.7 \%)\end{array}$ & $\begin{array}{c}50 \\
(27.7 \%)\end{array}$ & $\begin{array}{c}40 \\
(22.2 \%)\end{array}$ & $\begin{array}{c}62 \\
(34.4 \%)\end{array}$ & $\begin{array}{c}14 \\
(7.7 \%)\end{array}$ & \multirow{3}{*}{.000} \\
\hline & $\begin{array}{l}\text { Generic medicines are less effective compared to branded } \\
\text { medicines }\end{array}$ & $\begin{array}{l}\text { M.Pharm } \\
(\mathrm{N}=38)\end{array}$ & $\begin{array}{c}2 \\
(5.2 \%)\end{array}$ & $\begin{array}{c}6 \\
(15.7 \%)\end{array}$ & $\begin{array}{c}9 \\
(23.6 \%)\end{array}$ & $\begin{array}{c}18 \\
(47.6 \%)\end{array}$ & $\begin{array}{c}3 \\
(7 \%)\end{array}$ & \\
\hline & & $\begin{array}{l}\text { Pharm-D } \\
(\mathrm{N}=215)\end{array}$ & $\begin{array}{c}11 \\
(5.1 \%)\end{array}$ & $\begin{array}{c}38 \\
(17.6 \%)\end{array}$ & $\begin{array}{c}29 \\
(13.4 \%)\end{array}$ & $\begin{array}{c}96 \\
(44.6 \%)\end{array}$ & $\begin{array}{c}41 \\
(19 \%)\end{array}$ & \\
\hline \multirow{3}{*}{2} & & $\begin{array}{l}\text { B.Pharm } \\
(\mathrm{N}=180)\end{array}$ & $\begin{array}{c}7 \\
(3.8 \%)\end{array}$ & $\begin{array}{c}35 \\
(10.4 \%)\end{array}$ & $\begin{array}{c}53 \\
(29.4 \%)\end{array}$ & $\begin{array}{c}68 \\
(37.7 \%)\end{array}$ & $\begin{array}{c}17 \\
(9.4 \%)\end{array}$ & \multirow{3}{*}{.000} \\
\hline & $\begin{array}{c}\text { Generic medicines have higher adverse effects compared } \\
\text { to branded medicines }\end{array}$ & $\begin{array}{c}\text { M.Pharm } \\
(\mathrm{N}=38)\end{array}$ & $\begin{array}{c}2 \\
(5.2 \%)\end{array}$ & $\begin{array}{c}5 \\
(13.1 \%)\end{array}$ & $\begin{array}{c}5 \\
(13.1 \%)\end{array}$ & $\begin{array}{c}18 \\
(47.3 \%)\end{array}$ & $\begin{array}{c}8 \\
(21 \%)\end{array}$ & \\
\hline & & $\begin{array}{l}\text { Pharm-D } \\
(\mathrm{N}=215)\end{array}$ & $\begin{array}{c}6 \\
(2.7 \%)\end{array}$ & $\begin{array}{c}20 \\
(9.3 \%)\end{array}$ & $\begin{array}{c}50 \\
(23.2 \%)\end{array}$ & $\begin{array}{c}102 \\
(47.4 \%)\end{array}$ & $\begin{array}{c}37 \\
(17.2 \%)\end{array}$ & \\
\hline \multirow{3}{*}{3} & & $\begin{array}{l}\text { B.Pharm } \\
(\mathrm{N}=179)\end{array}$ & $\begin{array}{c}18 \\
(10 \%)\end{array}$ & $\begin{array}{c}69 \\
(38.5 \%)\end{array}$ & $\begin{array}{c}48 \\
(26.8 \%)\end{array}$ & $\begin{array}{c}35 \\
(19.5 \%)\end{array}$ & $\begin{array}{c}9 \\
(5 \%)\end{array}$ & \multirow{3}{*}{.506} \\
\hline & $\begin{array}{l}\text { Branded medicines are needed to have sophisticated } \\
\text { safety standards than generic medicines }\end{array}$ & $\begin{array}{l}\text { M.Pharm } \\
(\mathrm{N}=38)\end{array}$ & $\begin{array}{c}7 \\
(18.4 \%)\end{array}$ & $\begin{array}{c}9 \\
(23.6 \%)\end{array}$ & $\begin{array}{c}9 \\
(23.6 \%)\end{array}$ & $\begin{array}{c}11 \\
(28.9 \%)\end{array}$ & $\begin{array}{c}2 \\
(7.5 \%)\end{array}$ & \\
\hline & & $\begin{array}{l}\text { Pharm-D } \\
(\mathrm{N}=211)\end{array}$ & $\begin{array}{c}23 \\
(10.9 \%)\end{array}$ & $\begin{array}{c}69 \\
(32.7 \%)\end{array}$ & $\begin{array}{c}54 \\
(25.5 \%)\end{array}$ & $\begin{array}{c}49 \\
(23.2 \%)\end{array}$ & $\begin{array}{c}16 \\
(7.5 \%)\end{array}$ & \\
\hline \multirow{3}{*}{4} & & $\begin{array}{l}\text { B.Pharm } \\
(\mathrm{N}=181)\end{array}$ & $\begin{array}{c}10 \\
(5.8 \%)\end{array}$ & $\begin{array}{c}33 \\
(18.2 \%)\end{array}$ & $\begin{array}{c}45 \\
(24.6 \%)\end{array}$ & $\begin{array}{c}67 \\
(37 \%)\end{array}$ & $\begin{array}{c}26 \\
(14 \%)\end{array}$ & \multirow{3}{*}{.000} \\
\hline & Generic medicines are not as safe as branded drugs. & $\begin{array}{l}\text { M.Pharm } \\
(\mathrm{N}=38)\end{array}$ & $\begin{array}{c}0 \\
(0 \%)\end{array}$ & $\begin{array}{c}5 \\
(13.1 \%)\end{array}$ & $\begin{array}{c}5 \\
(13.1 \%)\end{array}$ & $\begin{array}{c}22 \\
(57.8 \%)\end{array}$ & $\begin{array}{c}6 \\
(15.7 \%)\end{array}$ & \\
\hline & & $\begin{array}{l}\text { Pharm-D } \\
(\mathrm{N}=216)\end{array}$ & $\begin{array}{c}4 \\
(1.8 \%)\end{array}$ & $\begin{array}{c}19 \\
(8.79 \%)\end{array}$ & $\begin{array}{c}35 \\
(16.2 \%)\end{array}$ & $\begin{array}{c}117 \\
(54.1 \%)\end{array}$ & $\begin{array}{c}41 \\
(18.9)\end{array}$ & \\
\hline \multirow{3}{*}{5} & & $\begin{array}{l}\text { B.Pharm } \\
(\mathrm{N}=181)\end{array}$ & $\begin{array}{c}10 \\
(5.5 \%)\end{array}$ & $\begin{array}{c}45 \\
(24.8 \%)\end{array}$ & $\begin{array}{c}65 \\
(35.9 \%)\end{array}$ & $\begin{array}{c}48 \\
(26.5 \%)\end{array}$ & $\begin{array}{c}13 \\
(7.1 \%)\end{array}$ & \multirow{3}{*}{.000} \\
\hline & Generic medicine requires more time to act in the body. & $\begin{array}{c}\text { M.Pharm } \\
(\mathrm{N}=38)\end{array}$ & $\begin{array}{c}0 \\
(0 \%)\end{array}$ & $\begin{array}{c}2 \\
(5.2 \%)\end{array}$ & $\begin{array}{c}10 \\
(26.3 \%)\end{array}$ & $\begin{array}{c}21 \\
(55.2 \%)\end{array}$ & $\begin{array}{c}5 \\
(14.2 \%)\end{array}$ & \\
\hline & & $\begin{array}{l}\text { Pharm-D } \\
(\mathrm{N}=216)\end{array}$ & $\begin{array}{c}8 \\
(3.7 \%)\end{array}$ & $\begin{array}{c}32 \\
(14.8 \%)\end{array}$ & $\begin{array}{c}16 \\
(7.4 \%)\end{array}$ & $\begin{array}{c}94 \\
(43.5 \%)\end{array}$ & $\begin{array}{c}38 \\
(17.5 \%)\end{array}$ & \\
\hline \multirow{3}{*}{6} & & $\begin{array}{l}\text { B.Pharm } \\
(\mathrm{N}=180)\end{array}$ & $\begin{array}{c}28 \\
(15.8 \%)\end{array}$ & $\begin{array}{c}67 \\
(37.2 \%)\end{array}$ & $\begin{array}{c}42 \\
(23.3 \%)\end{array}$ & $\begin{array}{c}31 \\
(17.2 \%)\end{array}$ & $\begin{array}{c}12 \\
(6.7 \%)\end{array}$ & \multirow{3}{*}{.000} \\
\hline & $\begin{array}{l}\text { A generic medicine is cheaper because they are } \\
\text { substandard to branded drugs. }\end{array}$ & $\begin{array}{c}\text { M.Pharm } \\
(\mathrm{N}=38)\end{array}$ & $\begin{array}{c}6 \\
(15.7 \%)\end{array}$ & $\begin{array}{c}10 \\
(26.3 \%)\end{array}$ & $\begin{array}{c}5 \\
(13.5 \%)\end{array}$ & $\begin{array}{c}11 \\
(28.9 \%)\end{array}$ & $\begin{array}{c}6 \\
(15.7 \%)\end{array}$ & \\
\hline & & $\begin{array}{r}\text { Pharm-D } \\
(\mathrm{N}=214)\end{array}$ & $\begin{array}{c}25 \\
(11.6 \%)\end{array}$ & $\begin{array}{c}56 \\
(26.1 \%)\end{array}$ & $\begin{array}{c}28 \\
(13 \%)\end{array}$ & $\begin{array}{c}67 \\
(31.3 \%)\end{array}$ & $\begin{array}{c}38 \\
(17.7 \%)\end{array}$ & \\
\hline
\end{tabular}

Kruskal-Wallis test $P$-value $<0.05^{*}, P$-value $<.01^{* *}$ and $P$-value $<.001^{* * *}$ is significant.

\section{DISCUSSION}

The main objective of this study is to compare the knowledge and perception about generic medicine among the students of B.Pharm, Mpharm, and Pharm.D. The current research could manifest unawareness regarding generic medication among B.Pharm and Pharm.D students compared to M.Pharm students, as more percentage of M.Pharm students compared to B.Pharm and Pharm.D correctly agreed that generic medicines are low-priced than branded ones and that the composition, in- dication, and dose of generic drugs are equal to branded medication. In comparison to their peers, M.Pharm students demonstrated explicit awareness of the bioequivalence feature of generic medications. This disparity in skill could be attributable to advanced biopharmaceutics being a subject in the M.Pharm curriculum, followed by Pharm.D, and finally B.Pharm. ${ }^{16}$

Our study could also assess the knowledge among M.Pharm, Pharm.D, and B.Pharm regarding the safety and efficacy of generic medicines. 


\section{Table 4: Perception of pharmacy cadres about generic medicine.}

\begin{tabular}{|c|c|c|c|c|c|c|c|c|}
\hline S. No & Questionnaire & Degree Enrolled & $\begin{array}{l}\text { Strongly } \\
\text { Agree } \\
\text { (\%) }\end{array}$ & $\begin{array}{l}\text { Agree } \\
(\%)\end{array}$ & $\begin{array}{l}\text { Neutral } \\
(\%)\end{array}$ & $\begin{array}{l}\text { Disagree } \\
\text { (\%) }\end{array}$ & $\begin{array}{l}\text { Strongly } \\
\text { Disagree } \\
\text { (\%) }\end{array}$ & $P$-value \\
\hline \multirow{3}{*}{1} & \multirow{3}{*}{$\begin{array}{l}\text { I think patients and doctors should be provided } \\
\text { sufficient knowledge regarding generic medicines to } \\
\text { assure they truly know them. }\end{array}$} & Bpharm $(\mathrm{N}=187)$ & $\begin{array}{c}75 \\
(40.1 \%)\end{array}$ & $\begin{array}{c}91 \\
(48.6 \%)\end{array}$ & $\begin{array}{c}14 \\
(7.4 \%)\end{array}$ & $\begin{array}{c}3 \\
(1.6 \%)\end{array}$ & $\begin{array}{c}4 \\
(2.1 \%)\end{array}$ & \multirow{3}{*}{.022} \\
\hline & & Mpharm (N=39) & $\begin{array}{c}21 \\
(53.8 \%)\end{array}$ & $\begin{array}{c}15 \\
(38.4 \%)\end{array}$ & $\begin{array}{c}2 \\
(5.1 \%)\end{array}$ & $\begin{array}{c}0 \\
(0 \%)\end{array}$ & $\begin{array}{c}1 \\
(2.56 \%)\end{array}$ & \\
\hline & & Pharm-D $(\mathrm{N}=218)$ & $\begin{array}{c}117 \\
(53.6 \%)\end{array}$ & $\begin{array}{c}82 \\
(37.6 \%)\end{array}$ & $\begin{array}{c}14 \\
(6.4 \%)\end{array}$ & $\begin{array}{c}2 \\
(0.9 \%)\end{array}$ & $\begin{array}{c}3 \\
(1.3 \%)\end{array}$ & \\
\hline \multirow{3}{*}{2} & \multirow{3}{*}{$\begin{array}{l}\text { I believe that advertisements by Pharmaceutical } \\
\text { companies persuade the usage of branded medicines. }\end{array}$} & Bpharm $(\mathrm{N}=189)$ & $\begin{array}{c}45 \\
(23.8 \%)\end{array}$ & $\begin{array}{c}102 \\
(53.9 \%)\end{array}$ & $\begin{array}{c}28 \\
(14.8 \%)\end{array}$ & $\begin{array}{c}9 \\
(4.7 \%)\end{array}$ & $\begin{array}{c}5 \\
(2.6 \%)\end{array}$ & \multirow{3}{*}{.000} \\
\hline & & Mpharm (N=39) & $\begin{array}{c}18 \\
(46.1 \%)\end{array}$ & $\begin{array}{c}17 \\
(43.59)\end{array}$ & $\begin{array}{c}3 \\
(7.6 \%)\end{array}$ & $\begin{array}{c}0 \\
(0 \%)\end{array}$ & $\begin{array}{c}1 \\
(2.5 \%)\end{array}$ & \\
\hline & & Pharm-D $(\mathrm{N}=219)$ & $\begin{array}{c}99 \\
(45.2 \%)\end{array}$ & $\begin{array}{c}97 \\
(44.2 \%)\end{array}$ & $\begin{array}{c}18 \\
(8.1 \%)\end{array}$ & $\begin{array}{c}5 \\
(2.2 \%)\end{array}$ & $\begin{array}{c}0 \\
(0 \%)\end{array}$ & \\
\hline \multirow{3}{*}{3} & \multirow{3}{*}{$\begin{array}{l}\text { Doctors and pharmacists should work together in } \\
\text { prescribing and dispensing drugs based on their } \\
\text { generic names and not brand names }\end{array}$} & Bpharm $(\mathrm{N}=189)$ & $\begin{array}{c}74 \\
(39.1 \%)\end{array}$ & $\begin{array}{c}81 \\
(42.8 \%)\end{array}$ & $\begin{array}{c}20 \\
(10.5 \%)\end{array}$ & $\begin{array}{c}8 \\
(4.2 \%)\end{array}$ & $\begin{array}{c}6 \\
(3.1 \%)\end{array}$ & \multirow{3}{*}{.000} \\
\hline & & Mpharm (N=39) & $\begin{array}{c}18 \\
(46.1 \%)\end{array}$ & $\begin{array}{c}18 \\
(46.1 \%)\end{array}$ & $\begin{array}{c}2 \\
(5.1 \%)\end{array}$ & $\begin{array}{c}1 \\
(2.8 \%)\end{array}$ & $\begin{array}{c}0 \\
(0 \%)\end{array}$ & \\
\hline & & Pharm-D $(\mathrm{N}=219)$ & $\begin{array}{c}129 \\
(58.9 \%)\end{array}$ & $\begin{array}{c}69 \\
(31.5 \%)\end{array}$ & $\begin{array}{c}13 \\
(5.9 \%)\end{array}$ & $\begin{array}{c}5 \\
(2.2 \%)\end{array}$ & $\begin{array}{c}3 \\
(1.3 \%)\end{array}$ & \\
\hline \multirow{3}{*}{4} & \multirow{3}{*}{$\begin{array}{l}\text { More information is required on the issues regarding } \\
\text { the safety and efficacy of generic medicines }\end{array}$} & Bpharm $(\mathrm{N}=186)$ & $\begin{array}{c}46 \\
(24.7 \%)\end{array}$ & $\begin{array}{c}105 \\
(56.4 \%)\end{array}$ & $\begin{array}{c}28 \\
(15 \%)\end{array}$ & $\begin{array}{c}6 \\
(3.2 \%)\end{array}$ & $\begin{array}{c}1 \\
(0.5 \%)\end{array}$ & \multirow{3}{*}{.022} \\
\hline & & Mpharm $(\mathrm{N}=39)$ & $\begin{array}{c}10 \\
(25.6 \%)\end{array}$ & $\begin{array}{c}22 \\
(56.4 \%)\end{array}$ & $\begin{array}{c}7 \\
(17.9 \%)\end{array}$ & $\begin{array}{c}0 \\
(0 \%)\end{array}$ & $\begin{array}{c}0 \\
(0 \%)\end{array}$ & \\
\hline & & Pharm-D $(\mathrm{N}=220)$ & $\begin{array}{c}84 \\
(38.1 \%)\end{array}$ & $\begin{array}{c}103 \\
(46.8 \%)\end{array}$ & $\begin{array}{c}25 \\
(11.3 \%)\end{array}$ & $\begin{array}{c}5 \\
(2.2)\end{array}$ & $\begin{array}{c}3 \\
(1.3 \%)\end{array}$ & \\
\hline \multirow{3}{*}{5} & \multirow{3}{*}{$\begin{array}{l}\text { It is easier to remember a medicine's therapeutic class } \\
\text { using generic names rather than brand names }\end{array}$} & Bpharm $(\mathrm{N}=188)$ & $\begin{array}{c}36 \\
(19.1 \%)\end{array}$ & $\begin{array}{c}83 \\
(44.1 \%)\end{array}$ & $\begin{array}{c}56 \\
(29.7 \%)\end{array}$ & $\begin{array}{c}10 \\
(5.3 \%)\end{array}$ & $\begin{array}{c}3 \\
(1.5 \%)\end{array}$ & \multirow{3}{*}{.000} \\
\hline & & Mpharm $(\mathrm{N}=39)$ & $\begin{array}{c}13 \\
(33.3 \%)\end{array}$ & $\begin{array}{c}21 \\
(53.8 \%)\end{array}$ & $\begin{array}{c}3 \\
(7.6 \%)\end{array}$ & $\begin{array}{c}2 \\
(5.1 \%)\end{array}$ & $\begin{array}{c}0 \\
(0 \%)\end{array}$ & \\
\hline & & Pharm-D $(\mathrm{N}=217)$ & $\begin{array}{c}117 \\
(53.9 \%)\end{array}$ & $\begin{array}{c}67 \\
(30.2 \%)\end{array}$ & $\begin{array}{c}28 \\
(12.9 \%)\end{array}$ & $\begin{array}{c}2 \\
(0.9 \%)\end{array}$ & $\begin{array}{c}3 \\
(1.3 \%)\end{array}$ & \\
\hline \multirow{3}{*}{6} & \multirow{3}{*}{$\begin{array}{l}\text { My pharmacy school education covers the topic of } \\
\text { cost-effective use of medicines very well. }\end{array}$} & Bpharm $(\mathrm{N}=189)$ & $\begin{array}{c}27 \\
(14.2 \%)\end{array}$ & $\begin{array}{c}96 \\
(50.7 \%)\end{array}$ & $\begin{array}{c}51 \\
(26.9 \%)\end{array}$ & $\begin{array}{c}11 \\
(5.8 \%)\end{array}$ & $\begin{array}{c}4 \\
(2.1 \%)\end{array}$ & \multirow{3}{*}{.000} \\
\hline & & Mpharm (N=39) & $\begin{array}{c}10 \\
(25.6 \%)\end{array}$ & $\begin{array}{c}17 \\
(43.5 \%)\end{array}$ & $\begin{array}{c}10 \\
(25.6 \%)\end{array}$ & $\begin{array}{c}1 \\
(2.5 \%)\end{array}$ & $\begin{array}{c}1 \\
(2.5 \%)\end{array}$ & \\
\hline & & Pharm-D $(\mathrm{N}=217)$ & $\begin{array}{c}75 \\
(34.5 \%)\end{array}$ & $\begin{array}{c}90 \\
(41.4 \%)\end{array}$ & $\begin{array}{c}40 \\
(18.4 \%)\end{array}$ & $\begin{array}{c}4 \\
(1.8 \%)\end{array}$ & $\begin{array}{c}8 \\
(3.6 \%)\end{array}$ & \\
\hline
\end{tabular}

Kruskal-Wallis test $P$-value $<0.05^{*}, P$-value $<.01^{* *}$ and $P$-value $<.001^{* * *}$ is significant.

A difference in learning among all the three Pharmacy cadres regarding this aspect could be found as Pharm.D students have more clinical exposure than Mpharm and B.Pharm students. Clinical exposure of Pharm.D students is attributable to their hospital postings as part of their practical training from the $2^{\text {nd }}$ year of their course. ${ }^{17}$

This research could identify better pharmacological knowledge about generic medicines among M.Pharm students than Pharm.D and B.Pharm students. More M.Pharm students disagreed that generic medicines take longer to act in the body than branded ones. M.Pharm students would be more acquainted with this topic as their syllabus covers biopharmaceutics in depth. Only $51 \%$ of M.Pharm, $46 \%$ of Pharm.D, and 38\% of B.Pharm disagree that brand name medicines require higher safety standards than generic medicines, which reveals a slight confusion regarding this concept, implying a lack of accurate information among the students.

Our study points out that, compared to B.Pharm students, more Pharm.D students think that patients and doctors need to be given adequate knowledge regarding generic medicines. Similarly, more than half of Pharm.D students, compared to their counterparts, strongly believe that pharmacists should work together in prescribing and dispensing drugs based on generic medicines and not brand names. They also find it easier to remember a medicine's therapeutic class using generic names rather than brand names. Ward rounds and working closely with doctors and patients had imbibed Pharm.D students to come to such conclusions pertinently. 
Chaudhary, et al:: Comparative Study of Generic Drugs among Pharmacy Cadres

More Pharm.D students compared to B.Pharm and M.Pharm strongly agree that their pharmacy school covers the topic of cost-effective use of medicines. However, for B.Pharm students, their course is devoid of sufficient clinical exposure and knowledge about pharmacoeconomics. Similarly, only less percentage of M.Pharm (pharmacy practice) student learns the subject. In contrast, Pharm.D education accentuates the principles of pharmacoeconomics in its syllabus. ${ }^{17-19}$ We also found out that an almost equal percentage of M.Pharm, Pharm.D, and B.Pharm students require additional information on the safety and efficacy of generic medicines.

This study had tried to evince the fact that the pharmacy students have a distended difference in the knowledge among the different cadres, which is shown in Table 2. In contrast, the cross-sectional study conducted in 2017 in Palestine among community pharmacists found no difference in the level of knowledge among the BSc Pharmacy, Pharm.D, M.Pharm, and $\mathrm{PhD}$ degree holders with the $P$-value of .975. The study also found no difference in the level of knowledge concerning their experience and practice with the $P$-value of .737 and .715 , respectively. ${ }^{19}$ The crosssectional study conducted in Qatar also found quite similar results as the survey done in Palestine. ${ }^{20,21}$

These findings reveal a pretty similar level of information about generic medicine among pharmacist professionals. However, there exists a difference in the level of knowledge among the students. Less exposure, practice, and training might have created such distinction, attributed to the difference in the curriculum of B.Pharm, Pharm.D, and M.Pharm.

\section{CONCLUSION}

Our study demonstrates a significant knowledge gap about generic medications among pharmacy cadres. A comparative analysis found that, as a post-graduate course, M.Pharm students appear to have a great understanding of the pharmacological characteristics of generic drugs. Whereas Pharm.D and B.Pharm students have a limited understanding of technical aspects of bioequivalence. B.Pharm is a degree program that emphasizes the manufacturing aspect of pharmaceuticals. Our study corroborates this assertion. Additionally, our survey demonstrates that, compared to other pharmacy students, most Pharm.D students possess adequate knowledge and apprehension regarding generic drugs and their significance in a hospital context.

When pharmacy students understand generic medicines and their use, they can assist in training and raising awareness about generic drugs among all cadres of the medical profession and the general public. This helps to persuade regulatory or government organizations to design and adopt robust generic drug policies, assisting in reducing the nation's health care expense.

\section{Limitation}

This study had developed a self-administered online form, so there are chances of incorrect filling, multiple fillings, and personal bias. This study had focused on the different cadres of pharmacy students of South India, especially Bangalore, so it can't be generalized for all of India. Furthermore, the study design used in this study was a cross-sectional study design, so it can't provide a cause-effect relationship.

\section{ACKNOWLEDGEMENT}

We are thankful to Dr. Shailesh Yadav for helping to carry out this study.

\section{CONFLICT OF INTEREST}

The authors declare no Conflict of interest.

\section{REFERENCES}

1. Mathew P. Generic drugs: review and experiences from South India. J Fam Med Prim Care. 2015;4(3):319-23. doi: 10.4103/2249-4863.161305, PMID 26286613.

2. Roy $V$, Gupta U, Agarwal AK. Cost of medicines and their affordability in private pharmacies in Delhi (India). Indian J Med Res. 2012;136(5):827-35. PMID 23287131.

3. James PB, Bah AJ, Margao EK, Hanson C, Kabba JA, Jamshed SQ. Exploring the knowledge and perception of generic medicines among final year undergraduate medical, pharmacy, and nursing students in Sierra Leone: A comparative cross-sectional approach. Pharmacy (Basel). 2018;6(1):3. doi: 10.3390/pharmacy6010003, PMID 29300350.

4. Mohammed AS, Woldekidan NA, Mohammed FA. Knowledge, attitude, and practice of pharmacy professionals on generic medicines in Eastern Ethiopia: A cross-sectional study. PLOS ONE. 2020;15(7):e0235205. doi: 10.1371/journal. pone.0235205, PMID 32658918.

5. Basak SC, Sathyanarayana D. Pharmacy education in India. Am J Pharm Educ. 2010;74(4):68. doi: 10.5688/aj740468, PMID 20585429.

6. Von Elm E, Altman DG, Egger M, Pocock SJ, Gøtzsche PC, Vandenbroucke JP, STROBE Initiative. The Strengthening the Reporting of Observational Studies in Epidemiology (STROBE) statement: guidelines for reporting observational studies. Bull World Health Organ. 2007;85(11):867-72. doi: 10.2471/blt.07.045120, PMID 18038077.

7. Available from: Strobe-statement.org. [Homepage on the internet]. STROBE statement: home [cited Sep 1 2020]. Available from: https://www.strobestatement.org/index.php?id=strobe-home.

8. Taherdoost $\mathrm{H}$. Validity and reliability of the research instrument; how to test the validation of a questionnaire/survey in a research. SSRN Journal. Aug 10 2016;2016. doi: 10.2139/ssrn.3205040.

9. Hajjar ST. Statistical analysis: internal-consistency reliability and construct validity. Int J Quant Qual Res Methods. 2018;6(1):27-38.

10. Schober $P$, Boer C, Schwarte LA. Correlation coefficients: appropriate use and interpretation. Anesth Analg. 2018;126(5):1763-8. doi: 10.1213/ ANE.0000000000002864, PMID 29481436

11. Raosoft. Com [homepage on the Internet]. Sample size Calculator by. Raosoft, Inc [cited Nov 10 2020]. Available from: http://www.raosoft.com/samplesize. html.

12. Wma.net. [Homepage on the Internet]. WMA-The World Medical AssociationWMA Declaration of Helsinki - ethical principles for medical research involving human subjects [cited Sep 1 2020]. Available from: https://www.wma.net/ policies-post/wma-declaration-of-helsinki-ethical-principles-for-medicalresearch-involving-human-subjects/.

13. Eysenbach G. Improving the quality of Web surveys: the Checklist for Reporting Results of Internet E-Surveys (CHERRIES). J Med Internet Res. 2004;6(3):e34 doi: 10.2196/jmir.6.3.e34, PMID 15471760

14. Microsoft. Com [homepage on the Internet]. Microsoft Excel Spreadsheet. Software|Microsoft 365

15. Ibm.com. [Homepage on the Internet]. SPSS Softw.

16. Pci. [Homepage on the Internet]; 2014. The master of pharmacy (M.Pharm) Course Regulations. Available from: nic.in [cited Oct 24 2020]. Available from: https://www.pci.nic.in/pdf/M.Pharm\%20Regulations.pdf.

17. Pci. [Homepage on the Internet]. Pharm.D. Regulations. 2008

18. Pci. [Homepage on the Internet]. The bachelor of pharmacy (B. Pham.) course regulations, 2014. Available from: nic.in [cited Oct 24 2020]. Available from: https://www.pci.nic.in/pdf/B.Pharm\%20Regulations.pdf.

19. Ahmad A, Patel I, Parimilakrishnan S, Mohanta GP, Chung H, Chang J. The role of pharmacoeconomics in current Indian healthcare system. J Res Pharm Pract. 2013;2(1):3-9. doi: 10.4103/2279-042X.114081, PMID 24991597.

20. Shraim NY, Al Taha TA, Qawasmeh RF, Jarrar HN, Shtaya MAN, Shayeb LA, Sweileh WM. Knowledge, attitudes and practices of community pharmacists on generic medicines in Palestine: a cross-sectional study. BMC Health Serv Res. 2017;17(1):847. doi: 10.1186/s12913-017-2813-z, PMID 29282055.

21. Awaisu A, Kheir N, Ibrahim MI, El-Hajj M, Hazi H, Khudair N, Barazi R. Knowledge, attitudes, and practices of community pharmacists on generic medicines in Qatar. Int J Clin Pharm. 2014;36(2):394-404. doi: 10.1007/s11096-013-9909-2, PMID 24532363 